\title{
Alpha-Fetoprotein-Producing Nonmetastatic Gastric Adenocarcinoma: A Rare Entity
}

\author{
A. Vivekanandarajah • J. P. Atallah • S. Gupta
}

Published online: 18 April 2013

(C) The Author(s) 2013. This article is published with open access at Springerlink.com

\section{Background}

Alpha-fetoprotein (AFP) is a plasma protein that is produced by the liver and the yolk sac during fetal development. In adults, an elevation in this protein can be exclusive to specific malignancies such as hepatocellular carcinoma, yolk sac tumors, and metastatic disease to the liver. In the clinical setting, an elevated AFP level and a liver mass (masses) translate to a diagnosis of hepatocellular carcinoma. Gastric adenocarcinoma is an aggressive malignancy that has a poor prognosis and has a potential for early metastatic spread [1]. There have been some case reports in the literature that have described elevated AFP levels in gastric adenocarcinoma with metastasis to the liver and hepatoid adenocarcinoma of the stomach. Here, we report an unusual case of nonmetastatic gastric adenocarcinoma with local compression on the liver and elevated AFP levels in a previously healthy woman.

\section{Case Presentation}

A 33-year-old woman from Saint Vincent Island presented to the emergency department with complaints of 6-month history of epigastric mass and pain, 15-lb weight loss, and poor appetite. She stated that she was evaluated by the local physician in the Caribbean and was told that she has a cyst.

\author{
A. Vivekanandarajah $(\varangle) \cdot$ J. P. Atallah $\cdot$ S. Gupta \\ Department of Hematology/Oncology, Staten Island University \\ Hospital, 475 Seaview Avenue, Staten Island, NY 10305, USA \\ e-mail: avivek27@gmail.com \\ J. P. Atallah \\ e-mail: jeanpaul_atallah@siuh.edu \\ S. Gupta \\ e-mail: drshilpigupta@gmail.com
}

No other further work-up was done. She denied fevers, chills, change in the color of the urine or stool, nausea, or vomiting. She was an ex-smoker and quit 10 years ago. She denied alcohol intake, any prescribed or illicit drug use, drug allergy, or any significant family history.

Vital signs revealed a pulse of 82 beats $/ \mathrm{min}$, blood pressure of $101 / 62 \mathrm{mmHg}$, respiratory rate of 20 respirations $/ \mathrm{min}$, and normal temperature. On physical exam, the patient appeared cachectic. Rest of the physical exam was significant for jaundice, scleral icterus, and a $14 \times 16 \mathrm{~cm}$ non-tender epigastric mass. Laboratory tests revealed the following: white blood count of $16,000 / \mu \mathrm{L}$, hemoglobin of $9.9 \mathrm{~g} / \mathrm{dL}$, platelet count of $882,000 / \mu \mathrm{L}$, total bilirubin of $8.7 \mathrm{mg} / \mathrm{dL}$, alkaline phosphatase of 590 units/L, albumin of $2.2 \mathrm{~g} / \mathrm{dL}$, LDH of 374 units/L, INR of 1.5, and normal glucose, electrolytes, renal function, amylase, lipase, and rest of the liver function tests. Tests of tumor markers revealed an AFP level of 1,451 ng/dL and normal carcinoembryonic antigen, CA 19-9, and BHCG. CT scan of the abdomen with oral and intravenous contrast revealed a heterogeneous upper abdominal mass measuring $12.6 \times 11.7 \times$ $11.4 \mathrm{~cm}$ inseparable from the lesser curvature of the stomach, compressing the liver hilum, periportal vessels and causing mild to moderate intrahepatic biliary ductal dilatation (Fig. 1). Ultrasound of the abdomen also confirmed these findings. Esophagogastroduodenoscopy revealed candidal esophagitis and a large smooth intramural or extrinsic mass arising from the lesser curvature of the stomach. Histopathological examination of the biopsy of the gastric mass was inconclusive. Histopathological examination and immunohistochemistry of CT-guided fine-needle aspiration of the abdominal mass revealed $\mathrm{CK}^{+}{ }^{+}$rare positivity, $\mathrm{CK} 20^{+}$, villin ${ }^{+}, \mathrm{CDX}-2^{+}$, and negative for CA 19-9 and CA 125. AFP stain was negative. These findings were consistent with upper gastrointestinal, pancreatobiliary, or bladder neoplasm. Ultrasound of the pelvis did not reveal any masses. Serology for Helicobacter pylori was positive, and treatment was initiated. She was also treated 


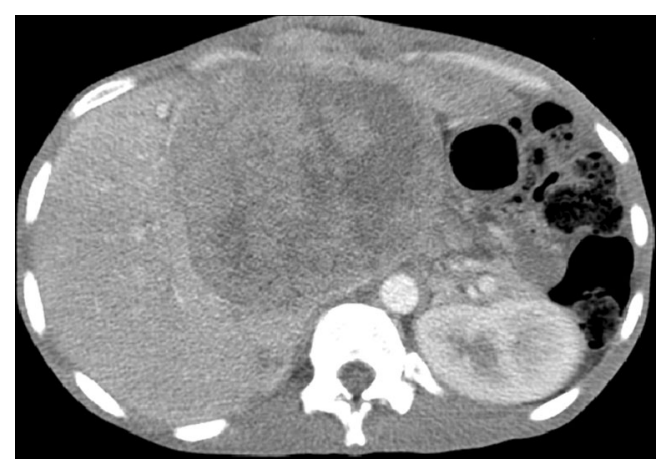

Fig. 1 Image presentation of heterogeneous upper abdominal mass

for candidal esophagitis. Further testing revealed sequential elevations in the total bilirubin, direct bilirubin, and the alkaline phosphatase levels. The patient became more cachectic and could not maintain adequate oral intake. Parenteral nutrition was started. She also underwent a laparoscopy and core biopsy of the abdominal mass, and the histopathological examination and immunohistochemistry revealed adenocarcinoma with extensive necrosis most consistent with upper gastrointestinal primary neoplasm. An independent external pathology laboratory also confirmed the same findings. Jejunostomy tube was also placed for feeding, and parenteral nutrition was discontinued. Due the rising bilirubin levels, a transhepatic cholangiogram was performed with placement of an internal/external biliary catheter for drainage. The bilirubin levels decreased to a nadir of $12.5 \mathrm{mg} / \mathrm{dL}$ after the placement of the biliary drainage catheter. Due to the abnormal bilirubin, the patient was not a candidate for the conventional gastric adenocarcinoma chemotherapy regimens, and the plan was to start chemotherapy consisting of weekly gemcitabine at a reduced dose to adjust for the bilirubin levels. She received gemcitabine at the reduced dose of $800 \mathrm{mg} / \mathrm{m}^{2}$. Pain control was achieved with intravenous hydromorphone via the patientcontrolled analgesia pump. Soon after the administration of chemotherapy, there was an improvement in the clinical and laboratory parameters. However, after a few days, there was deterioration in the patient's mental and respiratory status. CT scan of the head and the chest did not reveal metastasis or pulmonary embolism. Ammonia level was elevated, and lactulose was administered. She was not able to receive any further chemotherapy, and efforts were made to keep her on comfort care. Eventually, the patient was discharged in stable condition as per the family's wishes to the Saint Vincent Island.

\section{Discussion}

Gastric cancer is an aggressive malignancy with a poor prognosis. In the USA, gastric cancer ranks 14th in incidence among the other malignancies [2]. It is estimated that 21,320 people will be diagnosed with, and 10,540 people will die of gastric cancer in 2012 in the USA [2]. Adenocarcinoma is the most common histology and accounts for approximately 90 $95 \%$ of all gastric cancer. The average age of diagnosis is 70 , but the incidence of proximal gastric adenocarcinoma has risen dramatically in patients under the age of 40 over the last two decades [2]. Risk factors for gastric adenocarcinoma include advanced age, H. pylori infection, diets high in smoked or preserved meats, male gender, chronic atrophic gastritis, pernicious anemia, family history of gastric cancer, familial adenomatous polyposis, and history of gastric adenomatous polyps. Gastric cancer carries a dismal prognosis. Approximately $50 \%$ of patients can be cured with localized gastric cancer; however, it comprises only 10 to $20 \%$ of all cases diagnosed in the USA. Most patients have metastatic disease at the time of diagnosis. The 5 -year overall survival rate ranges from very minimal for patients with disseminated disease to almost $50 \%$ survival for patients with resectable localized distal gastric cancers. Even with apparent localized disease, the 5-year survival rate of patients with proximal gastric cancer is only 10 to $15 \%$.

In adults, AFP is a plasma protein produced mainly by hepatocellular carcinoma. Less commonly, AFP elevation can also be seen in other malignancies such as yolk sac tumor and hepatoid adenocarcinoma. High AFP levels have also been observed in gastric adenocarcinoma with synchronous hepatic metastasis. Among all GI malignancies, an elevation of the serum AFP level was reported in 1.3 to $15 \%$ of gastric cancers. Nonmetastatic gastric adenocarcinomas that secrete AFP are rare. Fifteen cases (3.9\%) out of 387 with gastric cancer showing elevation of AFP in serum was described in one of the reports in the literature; liver metastasis was observed in $12(80 \%)$ of these 15 cases [3]. AFP-producing gastric cancers can be divided into three subtypes: (1) hepatoid, (2) yolk sac tumor-like, and (3) fetal gastrointestinal [4]. AFP is most commonly produced by the hepatoid subtype. AFP-producing gastric cancers have a high proliferative activity, weak apoptosis, and rich neovascularization that render them very aggressive with a poor prognosis [5]. Recent reports have described that AFP-producing gastric tumors express specific receptors and factors associated with mitosis, cell movement, proliferative activity, and tumor progression such as Ki-67, hepatocyte growth factor and its receptor, cMet, vascular endothelial growth factor (VEGF), and its isoform VEGF-C which may contribute to the poor prognosis and drug resistance of these tumors [6, 7]. Benign conditions such as cirrhosis, biliary obstruction, pregnancy, and viral hepatitis can cause elevations in the serum AFP level. However, AFP values of greater than $500 \mathrm{ng} / \mathrm{mL}$ are unlikely due to benign conditions [8].

Limited successful treatment options exist for AFPproducing gastric cancer. Surgical resection is curative for localized tumors, and approximately $50 \%$ of patients who underwent curative resection of the tumor will relapse with 
hepatic metastasis [9]. These tumors respond poorly to a number of chemotherapy regimens. Some investigators have reported that AFP-producing gastric cancer can be treated successfully with neoadjuvant combination chemotherapy with epirubicin, fluorouracil (5-FU), and leucovorin [10]. Some authors have also reported the use of the FLEP regimen consisting of 5-FU, leucovorin, etoposide, and cisplatin for inoperable stage IV gastric cancer [11]. The FLEP chemotherapy regimen was more effective for stage IV AFP-producing gastric cancer than for stage IV non-AFP-producing gastric cancer, and it improved the prognosis of AFP-producing gastric cancer due to downstaging of the cancer. Other chemotherapy regimens consisting of 5-FU and paclitaxel have also been described [12]. Gemcitabine and cisplatin combination regimens have shown moderate efficacy in advanced gastric cancer [13].

\section{Conclusion}

Despite the utilization of aggressive multimodality treatment options, the prognosis for AFP-producing gastric cancers remains poor. Most patients who are diagnosed with AFPproducing gastric cancer have liver metastasis at the time of presentation. Our patient had AFP-producing gastric adenocarcinoma causing local compression on the biliary ducts, resulting in intrahepatic biliary dilatation and elevated AFP level. Repeat abdominal imaging did not reveal evidence of hepatic metastasis. It is possible that the patient could develop hepatic metastasis in the future. To the best of our knowledge, this is the first case report in the literature of an AFP-producing gastric adenocarcinoma without hepatic metastasis. We consider this case to be unique in terms of the rarity of presentation. Clinicians should be aware that although common tumors, such as the ones described above, produce AFP, gastric cancer in the nonmetastatic setting can also be a source of production of AFP.
Conflict of Interest The authors declare that they have no conflict of interest.

Open Access This article is distributed under the terms of the Creative Commons Attribution License which permits any use, distribution, and reproduction in any medium, provided the original author(s) and the source are credited.

\section{References}

1. Philip P, Ajani J. Gastric carcinoma. 2nd ed. Medical Oncology: A Comprehensive Review; 1997, TX.

2. Howlader N, Noone AM, Krapcho M, Neyman N, Aminou R, Altekruse SF et al. SEER Cancer Statistics Review 1975-2009.

3. Takahashi $\mathrm{Y}$ et al. Clinicopathological study of AFP producing gastric cancer-significance of AFP in gastric cancer. Nihon Geka Gakkai Zasshi. 1987;88(6):696-700.

4. Motoyama $\mathrm{T}$ et al. alpha-Fetoprotein producing gastric carcinomas: a comparative study of three different subtypes. Acta Pathol Jpn. 1993;43(11):654-61.

5. Koide $\mathrm{N}$ et al. Alpha-fetoprotein-producing gastric cancer: histochemical analysis of cell proliferation, apoptosis, and angiogenesis. Am J Gastroenterol. 1999;94(6):1658-63.

6. Amemiya $\mathrm{H}$ et al. High frequency of c-Met expression in gastric cancers producing alpha-fetoprotein. Oncology. 2000;59(2):145-51.

7. Kamei $\mathrm{S}$ et al. Evaluation of VEGF and VEGF-C expression in gastric cancer cells producing alpha-fetoprotein. J Gastroenterol. 2003;38(6):540-7.

8. van der Veek PP et al. Two patients with extremely elevated tumor markers: where is the malignancy? Gastroenterol Res Pract. 2011;2011:123743.

9. Inagawa $\mathrm{S}$ et al. Hepatoid adenocarcinoma of the stomach. Gastric Cancer. 2001;4(1):43-52.

10. Ihling $\mathrm{C}$ et al. Hepatoid adenocarcinoma of the stomach: a case report. Gen Diagn Pathol. 1995;141(1):61-5.

11. Kochi $M$ et al. FLEP chemotherapy for alpha-fetoproteinproducing gastric cancer. Oncology. 2004;66(6):445-9.

12. Takeyama $\mathrm{H}$ et al. Successful paclitaxel-based chemotherapy for an alpha-fetoprotein-producing gastric cancer patient with multiple liver metastases. World J Surg Oncol. 2007;5:79.

13. De Lange SM et al. Phase II trial of cisplatin and gemcitabine in patients with advanced gastric cancer. Ann Oncol. 2004;15(3):484-8. 